\title{
Assessment of Psychological Stress and Serum Cortisol in Patients having Chronic Periodontitis with and without Type II Diabetes Mellitus: A Clinicobiochemical Study
}

\author{
${ }^{1}$ Nilima S Rajhans, ${ }^{2}$ Girish Byakod, ${ }^{3}$ Radhika Kumar, ${ }^{4}$ Dhanesh Sable
}

\begin{abstract}
Background: The prevalence of periodontitis is significantly higher among people with poorly controlled diabetes mellitus (DM). The current study aimed to correlate the possible relation of stress in patients with chronic periodontitis as well as DM.

Materials and methods: $A$ total of 60 individuals were recruited in the age range of 35 to 50 years. Patients were divided into three groups which include 20 each: group I: chronic periodontitis with diabetic mellitus; group II: chronic periodontitis without DM; and group III: systemically and periodontally healthy individuals. Psychological assessment and biochemical analysis were carried out in all the patients.
\end{abstract}

Results: Mean clinical attachment level (CAL) in group I was $6.21 \mathrm{~mm}$, group II was $5.40 \mathrm{~mm}$, and group III was $0.00 \mathrm{~mm}$ (healthy periodontium). Group I recorded highest mean cortisol levels of 22.55, which was significantly higher as compared with group III, which presented a mean cortisol level of 12.72 . Mean cortisol level in group II was 15.70 , which was marginally higher than in group II. Individual cortisol levels were relatively constant in group II (minimum 14 and maximum 18). Group III presented a skewed individual cortisol levels (minimum 8.78 and maximum 17.31), whereas group I presented a significantly high individual cortisol levels (minimum 16 and maximum 25.5). Group III presented least mean prostate symptom score (PSS) of 6.75, group II 18.55, and group III 24.90.

Conclusion: There seems to be a strong correlation between periodontal destruction, DM stress, and serum cortisol levels.

Clinical significance: Psychosocial stress influences the course and progression of periodontitis and should be addressed before and during treatment.

Keywords: Cortisol, Diabetes, Periodontitis, Stress.

How to cite this article: Rajhans NS, Byakod G, Kumar R, Sable D. Assessment of Psychological Stress and Serum Cortisol in Patients having Chronic Periodontitis with and without

\footnotetext{
${ }^{1}$ Department of Periodontics, Saveeta University, Chennai, Tamil Nadu, India

2Department of Periodontics, M.A. Rangoonwala College of Dental Sciences \& Research Centre, Pune, Maharashtra, India

${ }^{3,4}$ Department of Periodontics, Shri Yashwantrao Chavan Memorial Medical \& Rural Development Foundation's Dental College \& Hospital, Ahmednagar, Maharashtra, India

Corresponding Author: Nilima S Rajhans, Department of Periodontics, Saveeta University, Chennai, Tamil Nadu, India Phone: +919860622850, e-mail: drshripadraj@gmail.com
}

Type II Diabetes Mellitus: A Clinicobiochemical Study. World J Dent 2017;8(5):378-381.

\section{Source of support: Nil}

Conflict of interest: None

\section{INTRODUCTION}

"A sound mind constitutes to a healthy body". This relationship has been recognized in history from the time of the ancient Romans, Greeks, and Chinese. In other words, a stress-free mind constitutes a healthy body.

$\mathrm{HSelye}^{1}$ has played a pivotal role in giving this term its current prominence. Selye coined the term "stress" and defined it as a reaction of the individual to forces acting concurrently on the body which if disproportionate, i.e., beyond straining capacity of adaptive process, lead to disease of exhaustion and death. ${ }^{2}$ He defined forces that challenge the adaptive competence of the individual as "stressors" and described that stressors could be mental or physical. He further elaborated that stressors producing positive changes in the body were termed as "eustress" and those stressors producing negative changes were termed as "distress". The individual's reaction to stress is termed as coping, and it refers to a person's efforts through action and thought to deal with demands perceived as taxing or overwhelming. ${ }^{3}$

It is entrenched that cardiovascular sickness, DM, preterm conveyance, osteoporosis, inflammatory bowel disease, rheumatoid joint pain, and systemic lupus erythematosus are identified with stress either as a physiological reaction to stress or as a behavioral reaction. It might be that stress is a huge normal hazard factor for DM, cardiovascular ailment, osteoporosis, preterm delivery, and in addition periodontal disorders. ${ }^{4}$ Obviously, extraordinary stressors and diverse reactions to stress might be agent in every illness. On the contrary or at the same time, push, i.e., altered by recognitions in adapting can offer ascent to well-being practices, which at that point could influence a similar range of perpetual maladies. The more serious episodes of every one of these conditions include initiation of the insusceptible reaction and a related increment in infection. ${ }^{5}$ 
Stress leads to downregulation of the immunity which is interceded principally through the hypothalamuspituitary-adrenal (HPA) and sympathetic adrenal medullary axes. The HPA and its final result, cortisol, is believed to be vital mediator of its connection between stressful life experience and well-being result. ${ }^{6}$ Further, the hormones secreted during a stressful event (glucagon, catecholamine, growth hormone, and cortisol) have shown to have a diabetogenic effect. The two-way relationship between diabetes and periodontal disorders is a welldocumented phenomenon. ${ }^{7}$

Hence, the goal of this study is to correlate the possible relation of stress in patients with chronic periodontitis as well as DM. A null hypothesis that "psychological stress and serum cortisol levels are not related to chronic periodontitis and diabetes mellitus" was put to test by this study.

\section{MATERIALS AND METHODS}

The current case-control study was conducted to evaluate psychological stress and serum cortisol levels in chronic periodontitis patients having DM. The study was approved by the Independent Ethics Committee and review board. A total of 60 individuals were recruited from the outpatient department of Shri. Yashwantrao Chavan Memorial Medical and Rural Development Foundation's Dental College and Hospital, Ahmednagar, India. The patients were in the age range of 35 to 50 years. All the patients were informed about the study protocol, and a written informed consent was obtained for participation in the study. Individuals were divided into three groups as follows:

Group I: 20 patients having chronic periodontitis with diabetic mellitus.

Group II: 20 patients having chronic periodontitis without DM.

Group III: 20 control group comprised of systemically and periodontally healthy individuals.

Criteria for chronic periodontitis were as laid down by the American Academy of Periodontology world workshop 1999. Individuals with any other known systemic diseases were excluded from the study. Pregnant women, individuals on any medication that can interfere with serum cortisol level or the periodontal status were also excluded from the study. Periodontal status was evaluated using plaque index (sillness and loe 1964), gingival index (loe and sillness 1963), probing pocket depth (PPD), and CAL. The periodontal examination was conducted using mouth mirror and Williams periodontal probe. The PPD and CAL were measured at six sites/tooth, i.e., mesiobuccal, midbuccal, distobuccal, mesiolingual, midlingual, and distolingual. All the existing teeth were included in the periodontal examination except root stumps and third molars.

\section{Psychological Assessment}

On the day of clinical examination, the stress analysis was performed using perceived stress scale by Sheldon cohen $^{8}$ and the social readjustment rating scale (SRRS) by Holmes and Rahe. ${ }^{9}$

The questionnaire was also translated to Marathi for easy understanding of the questions by the study participants.

\section{Biochemical Analysis}

A total of $3.5 \mathrm{~mL}$ of venous blood was collected from all participants after an overnight fast, in the morning between 8 and 10 am after 20 minutes of rest; $2 \mathrm{~mL}$ of blood sample was taken in fluoride oxalate containers for blood sugar level assessment, and $1.3 \mathrm{~mL}$ was taken into clean bulb for serum cortisol estimation. The fasting blood glucose levels were determined using glucose oxidase-peroxidase method.

\section{RESULTS}

Mean CAL in group I was $6.21 \mathrm{~mm}$ [standard deviation (SD) 0.797], group II was $5.40 \mathrm{~mm}$ (SD 0.575), and group III was $0.00 \mathrm{~mm}$ (healthy periodontium). Group I recorded highest mean cortisol levels of 22.55 (SD 2.77), which was significantly higher as compared with group III, which presented a mean cortisol level of 12.72 (SD 2.73). Mean cortisol level in group II was 15.70 (SD 1.21), which was marginally higher than group II. Individual cortisol levels were relatively constant in group II (minimum 14 and maximum 18). Group III presented a skewed individual cortisol levels (minimum 8.78 and maximum 17.31), whereas group I presented a significantly high individual cortisol levels (minimum 16 and maximum 25.5). Group III presented least mean PSS of 6.75, group II 18.55, and group III 24.90 (Tables 1 and 2).

\section{DISCUSSION}

Periodontal diseases are multifactorial and dynamic in nature where multiple factors engage in a variety of interactions. Onset and progression of periodontal diseases are clearly modified by local and systemic host conditions or risk factors, which markedly affect the resistance of the host to pathogenic organisms. Periodontal health is maintained by a delicate balance between host factors and microbial factors. Host factors are known to be altered by stress. Stress can have an immunosuppressive effect that can predispose an individual to periodontal disease. The studies have yielded positive correlative findings 
Table 1: Descriptive biochemical analysis in all the groups

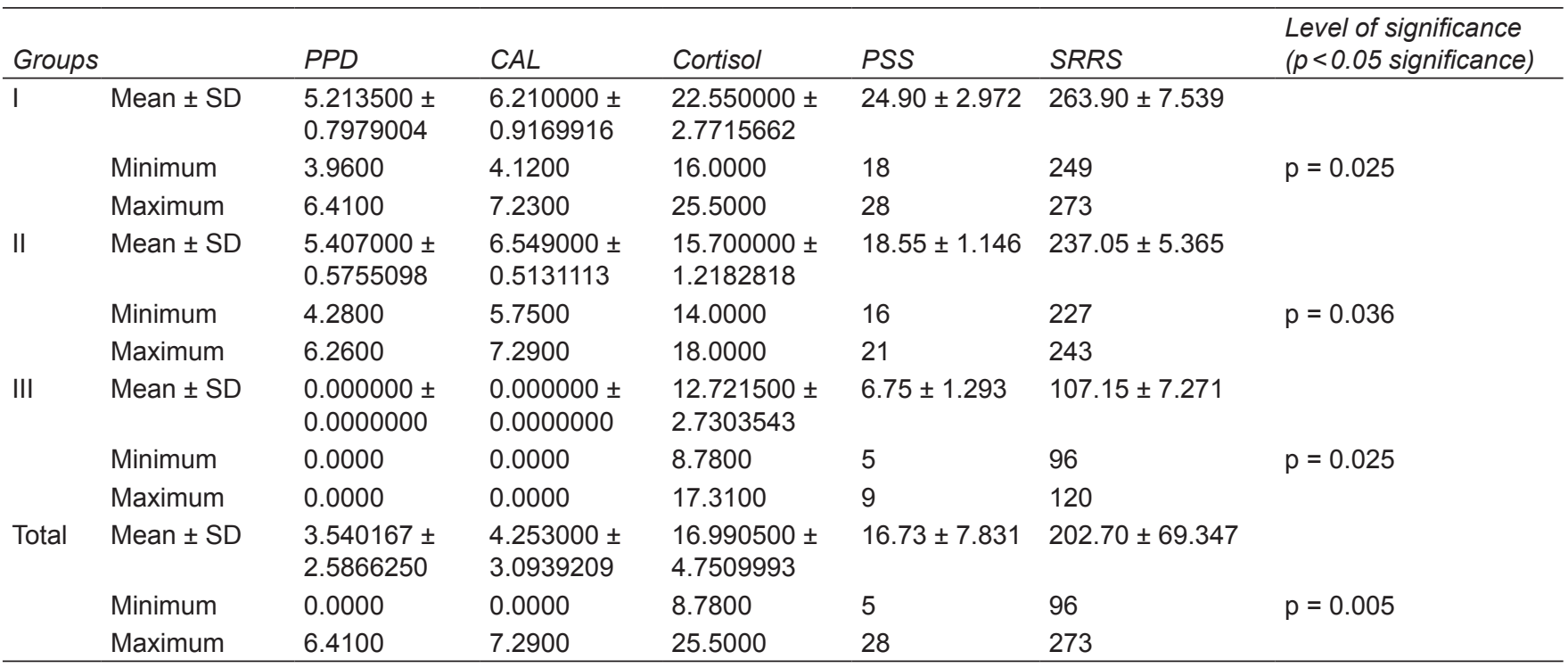

Table 2: Correlation among all the groups

\begin{tabular}{llll}
\hline & Group & Cortisol & SRRS \\
\hline Group & 1 & $-0.852^{* *}$ & $-0.931^{* *}$ \\
Cortisol & $-0.852^{* *}$ & 1 & $0.739^{* *}$ \\
SRRS & $-0.931^{* *}$ & $0.739^{* *}$ & 1 \\
\hline
\end{tabular}

**Significant correlation

between psychosocial factors and inflammatory periodontal diseases. ${ }^{10}$

In 1952, Albandar ${ }^{10}$ reported that severe cases of acute necrotizing ulcerative gingivitis were preceded by acute anxiety. Stress response represents a nonspecific reaction so that different types of stressors, each with their own unique properties, may operate through multiple stress pathways and yet produce common effects on immune system. Two main pathways link the brain and immune system - the HPA axis and direct neuronal fiber connections from the autonomic nervous system (ANS). Stress can also result in responses being transmitted to the ANS and then to adrenal medulla, resulting in secretion of catecholamines, such as epinephrine and norepinephrine. Catecholamines then affect prostaglandin and proteases, which in turn could enhance periodontal destruction. ${ }^{4}$ The present study showed a positive correlation between chronic periodontitis and psychological stress as indicated by PSS and SRRS (Table 1). Individuals with chronic periodontitis and DM showed significantly higher levels of serum cortisol as compared with other groups. Group I showed high levels of cortisol levels indicating higher stress levels. It is interesting to note that group I showed more periodontal destruction as compared with other two groups.

Association between periodontal disease and DM is well documented. Holzhausen et $\mathrm{al}^{11}$ observed that hyperglycemia may interfere with the periodontal disease because it leads to an exaggerated inflammatory response and impaired healing. The present study shows a significant correlation between high blood sugar levels and stress. Diabetes mellitus and stress both act synergistically leading to increased periodontal destruction.

Diabetic patients undergo a lot of emotional stress as well as physical stress as they go through a continuous process to alter their lifestyle and dietary habits. The present study indicated an increase in serum cortisol levels in DM, which in agreement with a study conducted in females by Hassan et al. ${ }^{12}$

The influence of cortisol in the glucose and lipid metabolism is in contrary to insulin. Hepatic glucose secretion is augmented, although insulin-stimulated glucose uptake in adipose tissue and muscle is disturbed. Elevated serum levels of cortisol of endogenous origin can cause insulin resistance and diabetes. Cortisol modifies blood glucose by disturbing glucose transporters in marginal tissues such as muscle and adipose tissue.

Prostate symptom score is a very good tool to assess psychological stress. It consists of 10 questions which are rated on 5 points from never (0) to almost always (4). Positively recorded items are reverse scored, and the ratings are summed. Highest score indicated highest risk. ${ }^{8}$ Social readjustment scale was developed in 1967 to identify chief traumatic life events. All the 43 stressful life events were regarded as a life-transforming unit depending on how distressing it was felt by a large number of subjects involved. Complete assessment of stressful life proceedings can be calculated by addition of scores for each episode practised over a 12-month duration. ${ }^{9}$

Diabetes mellitus and periodontal disease both can cause considerable anxiety in an individual, and the 
resultant stress makes the person more susceptible to tissue destruction. There was a significant increase in PPD and CAL in cases with higher SRRS and PSS. Periodontal destruction was also more in DM group. The studies demonstrated that psychosocial stress corresponds to a risk factor for periodontal disorder. ${ }^{4,13}$

\section{CONCLUSION}

From the results of this study, there seems to be a strong correlation between periodontal destruction, DM stress, and serum cortisol levels. However, as the present study design did not evade possible confounding factors, further research with a larger sample size aimed at a bigger demographic sample is needed.

\section{REFERENCES}

1. Selye, H. Selye's guide to stress research. New York: Van Nostrand Reinhold; 1936. pp. 246-257.

2. Sharma N, Gujjari SK, Kanagotaggi S. Stress and the periodontium. J Contemp Dent 2012 May-Aug;2(2):28-30.

3. Chandna S, Bathla M. Stress and periodontium: a review of concepts. J Oral Health Commun Dent 2010;4(Suppl 1): $17-22$.
4. Genco RJ, Ho AW, Kopman J, Grossi SG, Dunford RG, Tedesco LA. Models to evaluate the role of stress in periodontal disease. Ann Periodontol 1998 Jul;3(1):288-302.

5. Goyal S, Gupta G, Thomas B, Bhat KM, Bhat GS. Stress and periodontal disease: the link and logic!! Ind Psychiatry J 2013 Jan-Jun;22(1):4-11.

6. Chrousos GP. The hypothalamic-pituitary-adrenal axis and immune-mediated inflammation. N Engl J Med 1995 May;332(20):1351-1362.

7. Mealey BL. Periodontal disease and diabetes. a two-way street. J Am Dent Assoc 2006 Oct;137(Suppl):26S-31S.

8. Hildebrand HC, Epstein J, Larjava H. The influence of psychological stress on periodontal disease. J West Soc Periodontol Periodontal Abstr 2000; 48(3): 69-77.

9. Holmes $\mathrm{TH}$, Rahe $\mathrm{RH}$. The social readjustment rating scale. J Psychosom Res 1967 Aug;11(2):213-218.

10. Albandar JM. Epidemiology and risk factors of periodontal diseases. Dent Clin North Am 2005 Jul;49(3):517-532, v-vi.

11. Holzhausen M, Garcia DF, Pepato MT, Marcantonio E Jr. The influence of short-term diabetes mellitus and insulin therapy on alveolar bone loss in rats. J Periodontal Res 2004 Jul;39(3):188-193.

12. Hassan EAA, Ullah PHS, Al-Musawi A. Serum cortisol in Type 1 diabetic females. Diyala J Pure Sci 2012 Jan;8(1):e1-e6.

13. Mamza YP, Udoh AE, Etukudo MH. Evaluation of serum cortisol and growth hormone in Type 2 diabetic subjects attending University of maiduguri teaching hospital, Nigeria. IOSR J Dent Med Sci 2013 May-Jun;7(1):53-57. 